\title{
Implications for climate sensitivity from the response to individual forcings
}

Kate Marvel $^{1,2}$, Gavin A. Schmidt ${ }^{2}$, Ron L. Miller ${ }^{2}$, Larissa Nazarenko ${ }^{1,3}$

${ }^{1}$ Dept. of Applied Physics and Applied Mathematics, Columbia University, New York, USA

${ }^{2}$ NASA Goddard Institute for Space Studies, New York, USA

${ }^{3}$ Center for Climate Systems Research, Columbia University, New York

Climate sensitivity to doubled $\mathrm{CO}_{2}$ is a widely-used metric of the large-scale response to external forcing. Climate models predict a wide range for two commonly used definitions: the transient climate response (TCR: the warming after 70 years of $\mathrm{CO}_{2}$ concentrations that rise at $1 \%$ per year), and the equilibrium climate sensitivity (ECS: the equilibrium temperature change following a doubling of $\mathrm{CO}_{2}$ concentrations). Many observational datasets have been used to constrain these values, including temperature trends over the recent past ${ }^{1-6}$, inferences from paleo-climate ${ }^{7,8}$ and process-based constraints from the modern satellite era ${ }^{9,10}$. However, as the IPCC recently reported ${ }^{11}$, different classes of observational constraints produce somewhat incongruent ranges. Here we show that climate sensitivity estimates derived from recent observations must account for the efficacy of each forcing active during the historical period. When we use single forcing experiments to estimate these efficacies and calculate climate sensitivity from the observed twentieth-century warming, our estimates of both TCR and ECS are revised upward compared to previous studies, improving the consistency with independent constraints. 
The concept of radiative forcing is used to compare the effects of different physical drivers on the Earth's energy budget. By assumption, two forcing agents that produce a similar radiative imbalance would initiate similar feedbacks and have the same global mean temperature response ${ }^{12}$. However, there can be variations in the size and type of feedbacks engendered by a specific forcing $^{13}$, mainly due to geographical variations in the forcing magnitude. These variations can be characterized by an efficacy that scales for the differences in temperature response. Forcings that project more strongly on the Northern Hemisphere, land or polar regions are systematically more effective at changing temperatures than an equivalent amount of $\mathrm{CO}_{2}$, whose forcing is more uniformly distributed throughout the globe ${ }^{13,14}$. The converse is true for forcings localized in the Southern Hemisphere or ocean regions.

Some published constraints on ECS, particularly from the Last Glacial Maximum, have attempted to incorporate forcing efficacies into their assessments ${ }^{15,16}$, although none of the recently published constraints derived from modern trends have fully done $\mathrm{so}^{3-6}$. However, ECS does not provide the information on transient, short-term climate impacts that TCR reflects. It therefore remains unclear to what extent efficacies derived for equilibrium results are applicable to transient situations where ocean heat uptake plays an important role ${ }^{17-20}$.

An analysis of transient simulations with interactive aerosols ${ }^{21}$ indicated that the combination of anthropogenic aerosols, ozone, and land use change affect global temperature trends more efficiently than does $\mathrm{CO}_{2}$ forcing alone (i.e., the efficacy of the combination is greater than one). However, the specific contributions of individual forcings have thus far remained obscure. In this 
paper, we use a large suite of single-forcing simulations to estimate the impact of combined forcings in transient simulations for the historical period and show that proper consideration of the resulting efficacies implies that previously-derived constraints on ECS and TCR should be revised upward.

For the Coupled Model Intercomparison Project, Phase 5 (CMIP5), the NASA Goddard Institute for Space Studies (GISS) modeling group performed "historical" simulations using model version GISS-E2-R spanning 1850-2005 driven by estimates of relevant natural and external forcings $^{22}$. Multiple simulations over the same time period using single forcings or combinations of forcings were also submitted to the CMIP5 "historicalMisc" archive, including simulations forced by only well-mixed greenhouse gases (GHG), anthropogenic aerosols (AA), ozone $(\mathrm{Oz})$, solar variations $(\mathrm{Sl})$, volcanoes $(\mathrm{Vl})$, or land use changes (LU). These unique ensembles allow us to replicate climate sensitivity calculations in a "perfect model" framework, in which we have all the information we need to determine transient and equilibrium sensitivities using previously published methods, which can be compared to the actual TCR $\left(1.4^{\circ} \mathrm{C}\right)$ and $\mathrm{ECS}\left(2.3^{\circ} \mathrm{C}\right)$ of the GISS-E2-R model ${ }^{23,24}$.

TCR depends on the transient changes in global mean temperature $\Delta T$ and radiative forcing $\Delta F$. To calculate ECS, we also require estimates of the rate of ocean heat content (OHC) change $\Delta Q$. We use $\Delta Q$ here instead of the more conventional TOA imbalances ${ }^{25}$ in order to restrict our analysis to observables that have been used in previous analyses. Both $\Delta Q$ and $\Delta T$ are readily estimated from model output (see Methods). However, there are several different definitions of 
radiative forcing ${ }^{13,25}$, and we use two methods to capture different aspects of the planetary response to external agents. First, we calculate the annual-mean, global instantaneous radiative forcing (iRF) as the initial radiative flux change, evaluated at the tropopause in an attempt to anticipate the effect of rapid stratospheric adjustments ${ }^{26}$ for each year 1900-2005 (Methods). Second, we calculate the effective radiative forcing (ERF), which incorporates changes in the troposphere and land surface that are rapid compared to the ocean temperature response using fixed-SST experiments forced with year-2000 values of each forcing (Methods).

We first estimate the climate sensitivities using the instantaneous radiative forcings $\Delta F$, combined with the historical annual global mean temperature anomaly $\Delta T$ and ocean heat uptake anomaly $\Delta Q$ for each non-overlapping 10-year period beginning with 1906-1915 and ending with 1996-2005 (Methods, Figure S1).

For each decade, we plot the temperature anomaly versus forcing (for TCR, Fig. 1(a)) or the difference between forcing and ocean heat uptake anomalies (for ECS, Fig. 1(b)). Using

$$
\Delta F=\lambda_{T C R} \Delta T \quad ; \quad \Delta F=\lambda_{E C S} \Delta T+\Delta Q
$$

we calculate $\lambda$ as the slope of the best-fit line in both cases ${ }^{4}$. Using only the first and last decades gives comparable results. The TCR and ECS then given by

$$
T C R=\frac{F_{2 \times C O_{2}}}{\lambda_{T C R}} \quad ; \quad E C S=\frac{F_{2 \times C O_{2}}}{\lambda_{E C S}}
$$

where $F_{2 \times C O_{2}}=4.1 \mathrm{Wm}^{-2}$ is the model forcing (iRF) for $\mathrm{CO}_{2}$ doubling ${ }^{13}$. These linear methods assume that both $\lambda_{E C S}$ and $\lambda_{T C R}$ are constant in time, despite evidence ${ }^{27}$ that this may result in an underestimate of the "true" values. 
The ratios of single-forcing TCR and ECS to $\mathrm{CO}_{2}$-only TCR and ECS define transient and equilibrium efficacies, respectively ${ }^{13}$. These are measures of the enhancement (or suppression) of the climate response to the forcing relative to the climate response to greenhouse gases. Table S1 lists the transient and equilibrium efficacies calculated from the GISS-E2-R single-forcing runs, along with uncertainties derived from the 5-member ensembles for each forcing.

The global mean climate responses to different forcings may differ because of the character of the forcings themselves (such as their geographical or vertical distribution) and because different forcings induce different patterns of surface warming or cooling, thereby affecting the net top-ofatmosphere radiation imbalance, and thus the ocean heat uptake rate $\Delta Q$. The evolving pattern of temperature change may be incorporated into a global mean framework as an "ocean heat uptake efficacy" ${ }^{18}$. Our methodology does not differentiate between these two physical mechanisms, and we note that a substantial portion of what we call "forcing efficacy" may be due to differences between the ocean heat uptake induced by $\mathrm{CO}_{2}$ forcing and the heat uptake induced by the forcing in question.

In keeping with previous studies ${ }^{13,14,21,28}$, we find that aerosols have an enhanced transient climate response by roughly $30 \%$ and equilibrium response by $50 \%$. Additionally, the transient and equilibrium efficacies of ozone and volcanic forcing are significantly less than unity in the perfect model framework; other studies ${ }^{29,30}$ have also found that volcanic forcing has a smaller impact on global temperatures than an equivalent change in greenhouse gas forcing. The efficacies for LU and Sl calculated in this framework are uncertain due to the small changes in these forcing 
agents over the historical period, although we do find that the best estimates for LU transient and equilibrium efficacy generally exceed 1 , likely due to hemispheric asymmetry and land bias in this forcing.

Previous work ${ }^{13}$ found that certain equilibrium efficacies, notably anthropogenic tropospheric aerosols, decrease toward unity when the $\mathrm{iRF}$ is replaced by the ERF to incorporate tropospheric adjustments. This raises the possibility that the use of effective, rather than instantaneous radiative forcing, may render the sensitivities from GHG-only and historical simulations more directly comparable. We therefore re-calculate efficacies using the ERFs (open circles in Figs. 1 and S1). While aerosol transient and equilibrium efficacies are indeed reduced when using ERF (Table S1), TCR and ECS calculated from the combined effective radiative forcings within the "historical" experiment remain biased low compared to the GHG-only values (Fig. 1).

Since the forcings and temperature responses are additive, we can show in a vector plot the relative contributions of each forcing to the discrepancies between sensitivities derived from $\mathrm{CO}_{2}$-only simulations and those estimated from historical simulations (Fig. 1c-d). This shows clearly that the low sensitivities of the historical runs (compared to values obtained from $\mathrm{CO}_{2}$-only simulations) results from the higher efficacy of aerosols (when calculated using iRF) and land-use change, along with the lower efficacy of ozone and volcanic responses. Figs 1(c) and (d) indicate that many of the forcings over the recent historical period are less effective at changing global temperatures than those that cool the surface. We note that aerosol efficacy when calculated with ERF is compatible with unity; implying that differences between the historical sensitivities and 
$\mathrm{CO}_{2}$-only sensitivities are attributable to the other forcings.

Scaling $\Delta F$ for each of the single-forcing runs by the relevant efficacy yields sensitivities estimated from the historical runs comparable to those derived from $\mathrm{CO}_{2}$-only runs (Methods, Figure S2); since the forcings and temperatures add linearly, the resulting calculation with the historical all-forcing run scaled by the historical efficacy will also yield the same sensitivity as in the GHG-only runs.

What are the implications of our estimated forcing efficacies for constraints on sensitivity based on historical observations? Using our perfect model analysis, we can combine the model efficacies with historical forcings and the temperature response to estimate the observed climate sensitivity for comparison to existing calculations. Here, we make no attempt to evaluate the quality of existing observations or their suitability for estimating climate sensitivity; rather, we seek to replicate existing estimates and show how they change once efficacies are taken into account.

Assuming that all forcings have the same transient efficacy as greenhouse gases, and following a previous study ${ }^{4}$, the best estimate (median) for TCR is $1.3^{\circ} \mathrm{C}$ (see Methods). However, scaling each forcing by our estimates of transient efficacy (determined from either iRF or ERF), we obtain a best estimate for TCR of $1.8^{\circ} \mathrm{C}$ (Fig. 3(a)). This scaling simultaneously considers both forcing and ocean heat uptake efficacy. Other estimates of TCR ${ }^{3,21}$, which differ slightly due to choices of base period and uncertainty estimates and the aerosol forcing used, are similarly revised upward when using calculated efficacies (Fig. 3). 
We apply the same reasoning to estimates of ECS. Using an estimate ${ }^{4}$ of the rate of recent heat uptake $\Delta Q=0.65 \pm 0.27 \mathrm{~W} \mathrm{~m}^{-2}$, we find, assuming all equilibrium efficacies are unity, a best estimate of $\mathrm{ECS}=2.0^{\circ} \mathrm{C}$, comparable to the previous result $t^{4}$ of $1.9^{\circ} \mathrm{C}$. However, as with TCR, accounting for differences in equilibrium forcing efficacy revises the estimate upward; our new best estimate (using efficacies derived from the iRF) is $2.9^{\circ} \mathrm{C}$ (Fig. 2(a)). If efficacies are instead calculated from the ERF, the best estimate of ECS is $3.0^{\circ} \mathrm{C}$ (Fig. 2(b)). As for TCR, alternate estimates of ECS are revised upward when efficacies are taken into account (Fig. 2).

Estimates of both ECS and TCR are very sensitive to errors and uncertainties in the observations. Differences in the spread and best estimates for ECS and TCR (Table S2) will depend on the base periods used, estimates of ocean heat uptake, and on the aerosol forcing and its uncertainty. However, we note that in all cases, incorporating differing transient and equilibrium efficacies results in higher estimates for TCR and ECS.

The calculated efficacies used here are based on a single model. In order to increase confidence in these values it would be necessary to perform the suite of single-forcing experiments with additional models. These experiments were a low priority in CMIP5, and the historicalMisc archive is sparse. Moreover, very few groups performed comparable calculations of radiative forcings associated with each forcing agent. In cases where forcing is small over the whole historical period (LU, Sl) uncertainty is large. Simulations in which land use changes or solar forcings are amplified may be necessary to constrain the efficacy of these forcings.

We stress the importance of clearly defining "radiative forcing". While TCR and ECS esti- 
mates are revised upward regardless of the radiative forcing definition used to calculate efficacies, this is attributable to different factors when effective, rather than instantaneous, radiative forcing is used. The major difference is the calculated efficacy of anthropogenic aerosol forcing, which approaches unity when fast tropospheric adjustments are incorporated. Previous studies involving the GISS mode $1^{13}$ found that rapid cloud changes in both hemispheres result from the rapid adjustment to aerosol forcing; effective radiative forcing is thus more hemispherically symmetric than instantaneous aerosol forcing. This increased symmetry may account for the reduced aerosol efficacy when calculated with ERF. However, further study in a multi-model context will be necessary to better constrain the efficacy associated with historical aerosol changes.

GISS ModelE2 is more sensitive to $\mathrm{CO}_{2}$ alone than it is to the sum of the forcings that were important over the past century. This is largely a result of the low efficacy of ozone and volcanic forcings and the high efficacy of aerosol and LU forcing (which have had a cooling effect over the historical period), although further study is needed to explore model differences in simulating efficacies and to enhance confidence in these estimates. Climate sensitivities estimated from recent observations will therefore be biased low in comparison with $\mathrm{CO}_{2}$-only simulations due to an accident of history: when the efficacies of the forcings in the recent historical record are properly taken into account, estimates of TCR and ECS must be revised upward. Accounting for this results in recent historical estimates for TCR and ECS that are more consistent with constraints based on paleoclimate data and process-based constraints from modern climatology. Methodologies that attempt to combine independently-derived constraints on sensitivity should ensure that such biases are corrected before any synthesis is performed. 
1. Forest, C. E., Stone, P. H., Sokolov, A. P., Allen, M. R. \& Webster, M. D. Quantifying uncertainties in climate system properties with the use of recent climate observations. Science 295, 113-117 (2002).

2. Forest, C. E., Stone, P. H. \& Sokolov, A. P. Estimated pdfs of climate system properties including natural and anthropogenic forcings. Geophys. Res. Let. 33, doi:10.1029/2005GL023977 (2006).

3. Lewis, N. \& Curry, J. A. The implications for climate sensitivity of AR5 forcing and heat uptake estimates. Clim. Dyn. (2014).

4. Otto, A. et al. Energy budget constraints on climate response. Nature Geosci. 6, 415-416 (2013).

5. Ring, M. J., Lindner, D., Cross, E. F. \& Schlesinger, M. E. Causes of the global warming observed since the 19th century. ACS 02, 401-415 (2012).

6. Aldrin, M. et al. Bayesian estimation of climate sensitivity based on a simple climate model fitted to observations of hemispheric temperatures and global ocean heat content. Environmetrics 23, 253-271 (2012).

7. PALAEOSENS Project Members. Making sense of palaeoclimate sensitivity. Nature 491, 683-691 (2012).

8. Hargreaves, J. C., Annan, J. D., Yoshimori, M. \& Abe-Ouchi, A. Can the Last Glacial Maximum constrain climate sensitivity? Geophys. Res. Letts. 39 (2012). 
9. Sherwood, S. C., Bony, S. \& Dufresne, J. Spread in model climate sensitivity traced to atmospheric convective mixing. Nature 505, 37-42 (2014).

10. Fasullo, J. T. \& Trenberth, K. E. A less cloudy future: The role of subtropical subsidence in climate sensitivity. Science 338, 792-794 (2012).

11. Collins, M. et al. Long-term climate change: Projections, commitments and irreversibility. In Stocker, T. F. et al. (eds.) Climate Change 2013: The Physical Science Basis. Contribution of Working Group I to the Fifth Assessment Report of the Intergovernmental Panel on Climate Change (Cambridge University Press, Cambridge, United Kingdom and New York, NY, USA, 2013).

12. Hansen, J. et al. Climate sensitivity: Analysis of feedback mechanisms. In Hansen, J. E. \& Takahashi, T. (eds.) Climate Processes and Climate Sensitivity, no. 29 in AGU Geophys. Monograph, 130-163 (American Geophysical Union, Washington, D. C., 1984).

13. Hansen, J. E. et al. Efficacy of climate forcings. J. Geophys. Res. 110 (2005).

14. Stuber, N., Ponater, M. \& Sausen, R. Why radiative forcing might fail as a predictor of climate change. Climate dynamics 24, 497-510 (2005).

15. Köhler, P. et al. What caused Earth's temperature variations during the last 800,000 years? Data-based evidence on radiative forcing and constraints on climate sensitivity. Quat. Sci. Revs. 29, 129-145 (2010).

16. Schmittner, A. et al. Climate sensitivity estimated from temperature reconstructions of the Last Glacial Maximum. Science 334, 1385-1388 (2011). 
17. Rose, B. E., Armour, K. C., Battisti, D. S., Feldl, N. \& Koll, D. D. The dependence of transient climate sensitivity and radiative feedbacks on the spatial pattern of ocean heat uptake. Geophysical Research Letters 41, 1071-1078 (2014).

18. Winton, M., Takahashi, K. \& Held, I. M. Importance of ocean heat uptake efficacy to transient climate change. Journal of Climate 23, 2333-2344 (2010).

19. Armour, K. C., Bitz, C. M. \& Roe, G. H. Time-varying climate sensitivity from regional feedbacks. J. Clim. 26, 4518-4534 (2013).

20. Kummer, J. R. \& Dessler, A. E. The impact of forcing efficacy on the equilibrium climate sensitivity. Geophys. Res. Letts. 41, 3565-3568 (2014).

21. Shindell, D. T. Inhomogeneous forcing and transient climate sensitivity. Nature Climate Change 4, 274-277 (2014).

22. Miller, R. L. et al. CMIP5 historical simulations (1850-2012) with GISS ModelE2. J. Adv. Model. Earth Syst. 6, 441-477 (2014).

23. Nazarenko, L. et al. Future climate change under rcp emission scenarios with giss modele2. Journal of Advances in Modeling Earth Systems 7, 244-267 (2015).

24. Schmidt, G. A. et al. Configuration and assessment of the GISS ModelE2 contributions to the CMIP5 archive. J. Adv. Model. Earth Syst. 6, 141-184 (2014).

25. Gregory, J. M. et al. A new method for diagnosing radiative forcing and climate sensitivity. Geophys. Res. Lett. 31 (2004). 
26. Hansen, J. E. et al. Forcings and chaos in interannual to decadal climate change. J. Geophys. Res. 102, 25,679-25,720 (1997).

27. Gregory, J. M., Andrews, T. \& Good, P. The inconstancy of the transient climate response parameter under increasing co2. Philos. Trans. R. Soc. London in press (2015).

28. Shindell, D. \& Faluvegi, G. Climate response to regional radiative forcing during the twentieth century. Nature Geoscience 2, 294-300 (2009).

29. Tomassini, L., Reichert, P., Knutti, R., Stocker, T. F. \& Borsuk, M. E. Robust bayesian uncertainty analysis of climate system properties using Markov Chain Monte Carlo methods. $J$. Climate 20, 1239-1254 (2007).

30. Merlis, T. M., Held, I. M., Stenchikov, G. L., Zeng, F. \& Horowitz, L. W. Constraining transient climate sensitivity using coupled climate model simulations of volcanic eruptions. Journal of Climate 27, 7781-7795 (2014).

Acknowledgements Climate modeling at GISS is supported by the NASA Modeling, Analysis and Prediction program and resources supporting this work were provided by the NASA High-End Computing (HEC) Program through the NASA Center for Climate Simulation (NCCS) at Goddard Space Flight Center.

Competing Interests The authors declare that they have no competing financial interests.

Correspondence Correspondence and requests for materials should be addressed to Kate Marvel (email: kate.marvel@ nasa.gov). 
Author contributions KM and GS designed the research and wrote the paper, with input from RM. RM and LN provided the forcing data. LN ran the climate model experiments. All authors contributed to the interpretation of the results.
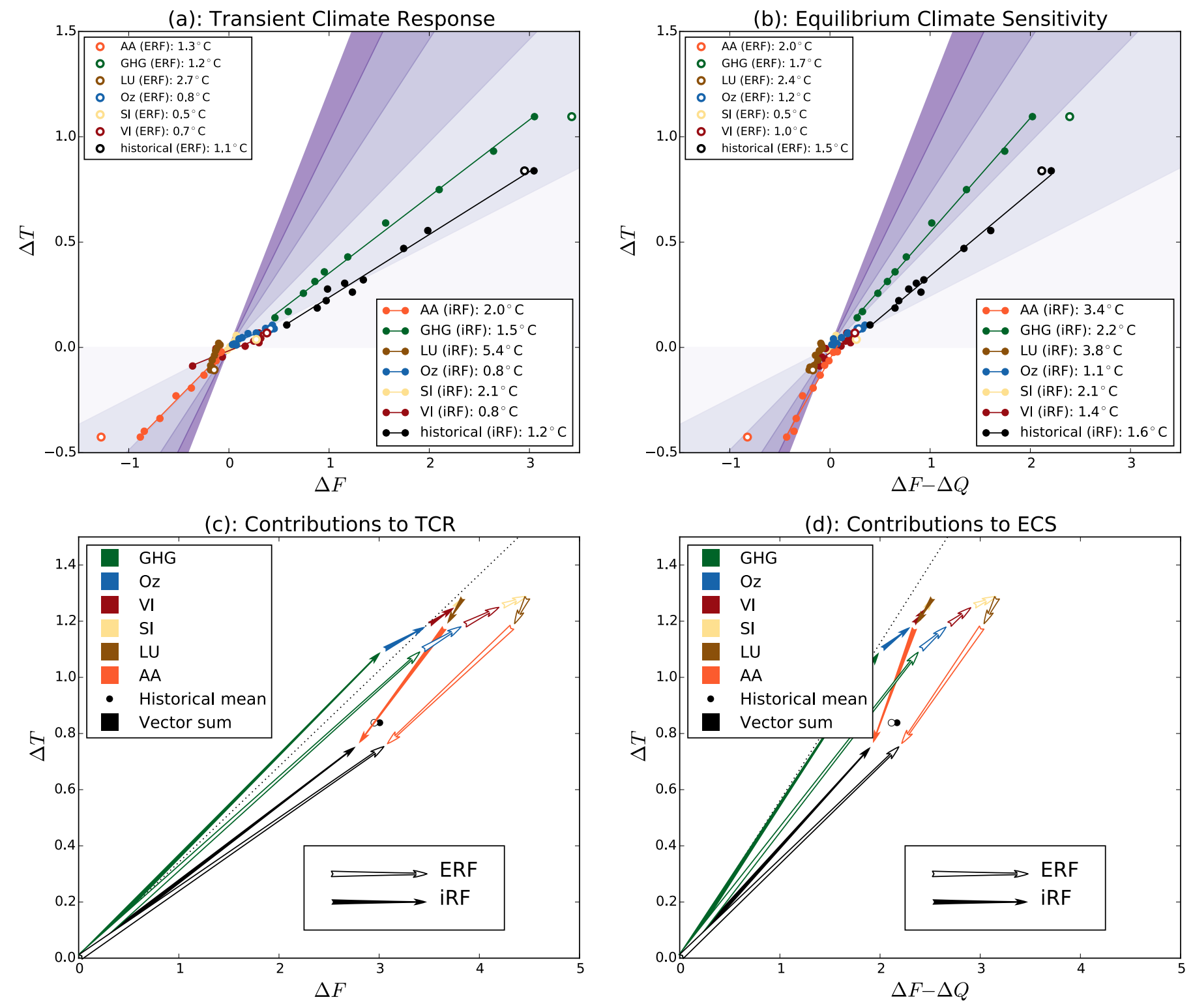
(a): Efficacies from instantaneous RF

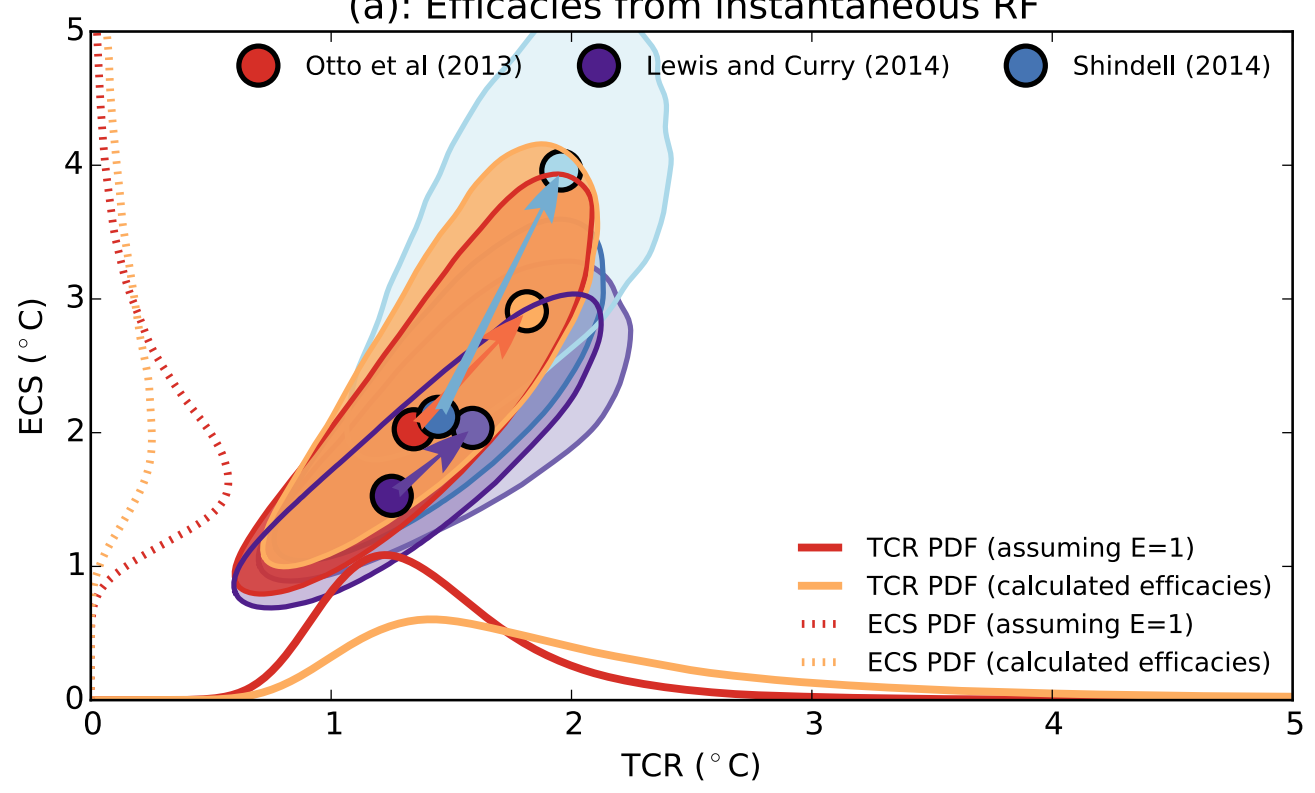

(b): Efficacies from effective RF

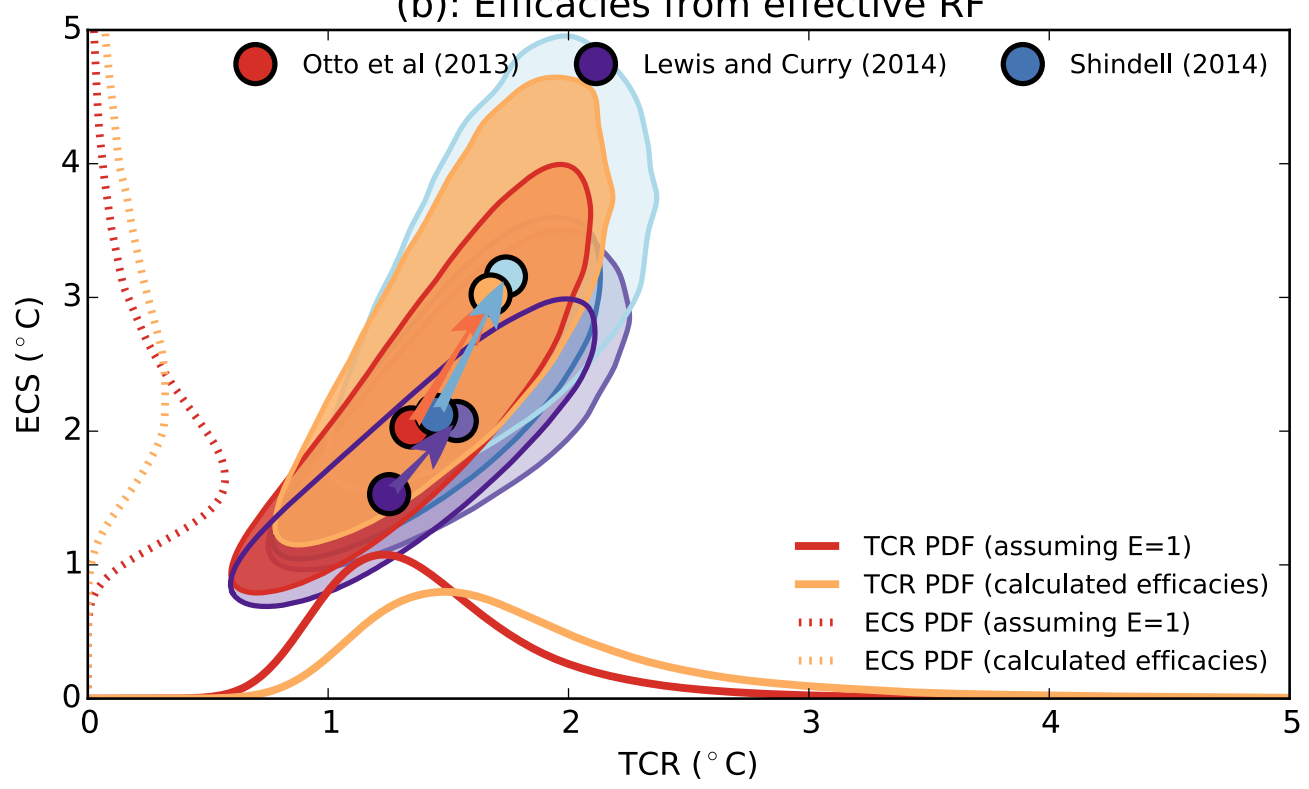


Figure 1 (a) Non-overlapping ensemble average decadal mean changes in temperature and instantaneous radiative forcing for GISS-E2-R single-forcing ensembles (filled circles). TCR is calculated from the slope of the best-fit line. Also shown are 1996-2005 temperature changes and effective radiative forcing (open circles). In this case, TCR is the quotient of the temperature and ERF estimates. Following ${ }^{4}$, straight purple contours show isolines of TCR from 0 to 4 (b) Same, but changes in the rate of ocean heat uptake are subtracted from forcing changes. ECS is calculated from the slope of the best-fit line (for iRF) or from the quotient (for ERF). (c) 1996-2005 average $\Delta T$ and instantaneous (filled arrows) and effective (white arrows) radiative forcing for each single-forcing experiment. The transient climate response for each experiment in each case is the slope of the line. The vector sum of the single-forcing values does not substantially differ from the historical values (circles) and the TCR of the sum and historical experiments is less than that of the GHG-only experiment. The published GISS-E2-R TCR $\left(1.4^{\circ} \mathrm{C}\right)$ is shown as a dashed black line. (d) Same as (c), but the x-axis shows the difference of 1996-2005 average forcing and estimated ocean heat uptake. The slope of each line is the equilibrium climate sensitivity. The published GISS-E2-R ECS $\left(2.3^{\circ} \mathrm{C}\right)$ is shown as a dashed black line.

Figure 2 (a) TCR ( $x$-axis) and ECS ( $y$-axis) best-guess values (circles) and 95\% joint confidence intervals (shaded regions). These are first calculated assuming all efficacies $=$ 1 (darker colors) and then incorporating efficacies calculated using instantaneous radiative forcing (iRF). Arrows indicate the revisions in TCR and ECS when efficacies are taken 
into account. Estimates from three published works ${ }^{3,4,21}$ and their revisions are shown. Original (red) and revised (orange) TCR and ECS probability distribution functions calculated using Otto et al. data are shown on the $x$ - and $y$-axes, respectively. (b) Same, but efficacies are calculated using effective radiative forcing (ERF). 


\title{
METHODS: Implications for climate sensitivity from the response to individual forcings
}

\author{
Kate Marvel ${ }^{1,2}$, Gavin A. Schmidt ${ }^{2}$, Ron L. Miller ${ }^{2}$, \\ Larissa Nazarenko ${ }^{1,3}$
}

October 1, 2015

\section{Simulations}

We use a large suite of historical simulations from the GISS-E2-R model[12] with multiple subsets of relevant forcings as archived in the CMIP5 database. Specifically, we use a 6-member ensemble of simulations with "historical" forcings including well-mixed greenhouse gases, anthropogenic aerosols, land use/land cover change, ozone changes, and volcanic and solar forcing[8]. Additionally, we use 5-member ensembles with each of the forcings run separately ("historicalMisc" simulations).

The model consists of GISS ModelE2 for the atmosphere coupled to the Russell ocean model. All simulations use physics version 1 (Non-Interactive atmospheric composition - NINT), in which aerosols and ozone are read in via pre-computed transient aerosol and ozone fields. The aerosol indirect effect is parameterized. Further information on the model configuration and specific experiments can be found online at http://data.giss . nasa.gov/modelE/ar5/.

\section{Perfect Model Framework}

The GISS model output provides all the diagnostics necessary to determine the transient climate response and equilibrium climate sensitivity for each singleforcing ensemble and for the "historical" ensemble using previously established methodologies. Surface air temperatures and ocean potential temperatures are available as standard CMIP5 output. Because radiative forcing calculations were 
not required in CMIP5, we have made iRF time series and ERF values for the GISS model simulations available on the GISS website at http://data.giss . nasa.gov/modelforce/.

In this section, we describe the calculations performed in order to obtain $\Delta T, \Delta F$, and $\Delta Q$ from the GISS-E2-R simulations. The relevant time series and values are plotted in Figure $S 1$, and described in further detail below.

\subsection{Instantaneous radiative forcing (iRF) definition}

We calculate the TOA radiative forcings associated with each climate driver using a radiation-only calculation for each year between 1851 and 2005 with the driver changing, but with all other variables set at pre-industrial (1850) values. In each case, we approximate the effect of rapid stratospheric adjustment by evaluating the forcing at the tropopause instead of top of atmosphere $[6,8$,$] . The 10$-year running means of iRF for each single-forcing ensemble are plotted as dashed lines in Figure $\mathrm{S} 1(\mathrm{a})-(\mathrm{g})$.

\subsection{Effective radiative forcing (ERF) definition}

The effective radiative forcing is calculated from climate model runs in which the forcing is held constant at year 2000 values and SSTs are fixed at their 1850 values. Following [4], we define effective radiative forcing as

$$
E R F=F_{o}+\Delta T_{a} / \lambda
$$

where $F_{o}$ is the flux change at the top of the atmosphere, $\Delta T_{a}$ the global surface air temperature change with SSTs fixed at pre-industrial values (i.e., as they were in 1850), and $\lambda$ taken from previously published GISS-E2-R simulations. This technically assumes unit efficacy for all forcings, but the response term $\Delta T_{a} / \lambda$ is in general small compared to the TOA flux change. All values are ten-year means. When ERF is used, TCR and ECS are calculated as the quotient of 1996-2005 average $\Delta T$ and year-2000 ERF and year-2000 ERF minus 1996-2005 trends in ocean heat uptake, respectively. The calculated ERF 10-year averages centered on the year 2000 are shown as unfilled dots in Figure S1(a)-(g). We obtain similar results when ERF is calculated using 30-year averages. 
(a): historical

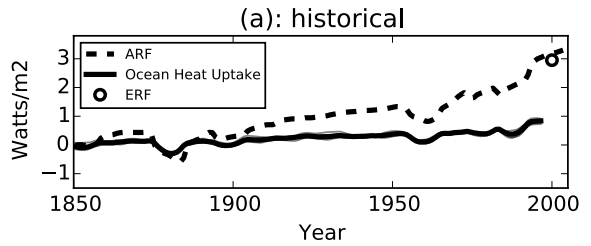

(c): SI

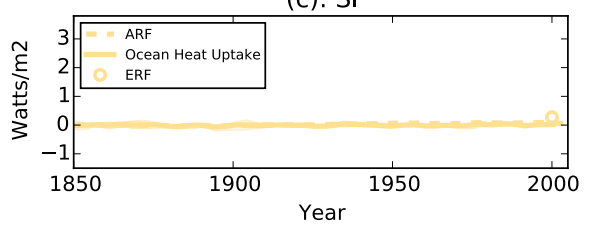

(e): Oz

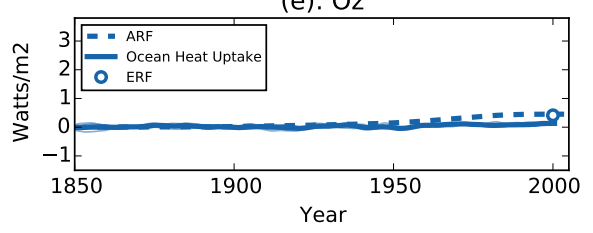

(g): LU

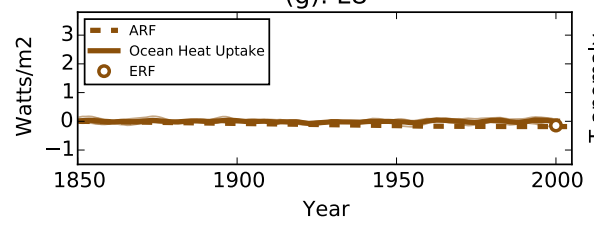

(b): GHG

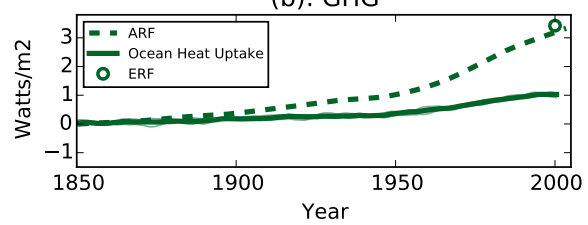

(d): VI

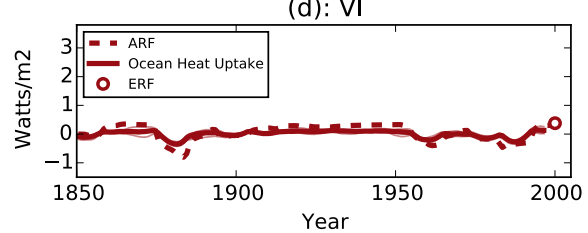

(f): AA

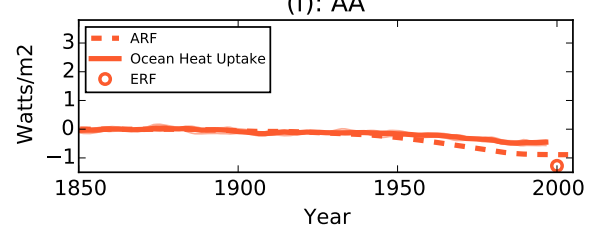

(h): Temperatures

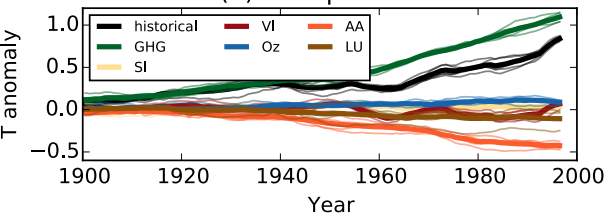

Figure S1: (a-g): Ensemble-average instantaneous radiative forcings and ocean heat uptake rates (thick lines) and individual ensemble members (thin) for GISSE2-R single-forcing experiments. All quantities are 10-year running means. Dots represent year-2000 effective radiative forcings (ERF). (h): Ensemble-average temperature anomalies (relative to 1850) for each single-forcing simulation. 


\subsection{Model ocean heat content}

As in Palmer et al.[11], we calculate the ocean heat content (OHC) for every simulation using

$$
\Phi=\sum_{i, j, k} \rho C \theta_{i, j, k} A_{i, j} d z_{k}
$$

where $\rho=3985 \mathrm{~J} \mathrm{~kg}^{-1} \mathrm{~K}^{-1}$ is the specific heat content, $\rho=1025 \mathrm{~kg} \mathrm{~m}^{-3}$ the density of seawater, $\theta$ is the annual mean ocean potential temperature, and $A_{i, j}$ the area of the grid cell. Because the Russell ocean model is mass-conserving, we multiply the area by the varying vertical layer thickness $d z$.

The single-forcing experiments are spun off from a long pre-industrial control run, with the first ensemble member branching after 3981 years of integration and subsequent members branching at 20 -year intervals. To account for a small residual control run drift, we subtract the linear trend in the relevant control run time period from each ensemble member, thereby calculating an anomaly time series relative to the pre-industrial period. Alternate methods to assess drift (such as a loess fit) make no significant difference to our results. We estimate the decadal rate of ocean heat uptake by calculating the best-fit linear trend to 10 -year segments of OHC. Ensemble average ocean heat uptake rates for each single-forcing simulation are shown in Figure $\mathrm{S} 1(\mathrm{a})-(\mathrm{g})$ as solid lines.

\subsection{Temperature anomalies}

For each simulation, we calculate global-average, annual-average temperature anomalies with respect to pre-industrial control averages. Any temperature drift in the pre-industrial control run is removed using the same procedure as used for ocean heat content. These time series are plotted in Figure S1(h).

\subsection{Calculating ECS and TCR}

As discussed in the main text, we calculate ECS and TCR using

$$
\Delta F=\lambda_{T C R} \Delta T \quad ; \quad \Delta F=\lambda_{E C S} \Delta T+\Delta Q .
$$

This framework is similar to that used in previous studies [2]. Our $\lambda_{E C S}$ is equivalent to a "climate response parameter" (called $\alpha$ in Gregory et. al.) that measures the overall feedback strength of the climate system. The transient parameter $\lambda_{T C R}$ measures the "climate resistance" [3], or the sum of $\lambda_{E C S}$ and an ocean heat uptake efficacy $\kappa$, if it is assumed that $\Delta Q=\kappa \Delta T$. 
We note that there are significant complications inherent in estimating both ECS and TCR from these transient single-forcing or historical simulations. Numerous studies (e.g. $[14,1,5]$ ) suggest that the net radiation lost to space is a function of the surface temperature pattern, itself largely related to geographical variations in ocean heat uptake. Winton et al[14] suggest that this may be simply incorporated into the global mean framework by multiplying $\Delta Q$ by an ocean heat uptake efficacy factor $\epsilon$. This factor, which should not be confused with the ocean heat uptake efficiency $\kappa$, reflects the changing relationship between global mean $\Delta T$ and outgoing radiation [5] as the surface warming pattern evolves, and is shown[14] to be greater than unity in most models. Thus, our sensitivity estimates are likely to underestimate the "true" values, even when forcing efficacy is taken into account. We retain these definitions, however, for consistency with earlier literature estimating ECS and TCR from historical observations $[10,7,13]$.

\section{Efficacies}

The efficacy of a particular driver is calculated from 5-member ensembles (6 in the "historical" case) forced only with that driver or collection of drivers. We calculate the TCR (from temperature and forcing changes relative to preindustrial control) and ECS (from temperature, forcing, and ocean heat content changes) as described in the main text. Transient and equilibrium efficacies $E_{i}$ are defined as the quotient of the TCR or ECS calculated from a model run with forcing $i$ and the relevant previously published GISS-E2-R TCR and ECS values $\left(1.4^{\circ} \mathrm{C}\right.$ and $2.3^{\circ} \mathrm{C}$ respectively).

In the iRF case, where annual forcing time series are available, TCR and ECS are calculated by regressing ensemble-average decadal mean forcing or forcing minus ocean heat content change rate against ensemble-average temperature change. We assume that efficacies remain roughly constant in time over the historical period, an assumption bolstered by the high temporal correlation between ensemble average decadal mean temperature and forcing changes (with correlation coefficient over .99 for each single-forcing experiment). In the ERF case, we have only one decade available; hence efficacies are estimated using the quotient of temperature change and forcing and/or $\mathrm{OHC}$ uptake changes.

The uncertainty in the efficacies is estimated from individual members of the single-forcing ensembles (Figure S2). Confidence intervals on the sample mean are constructed using a student-t distribution with 4 degrees of freedom ( 5 in the case of the 6-member historical ensemble). 
(a): iRF
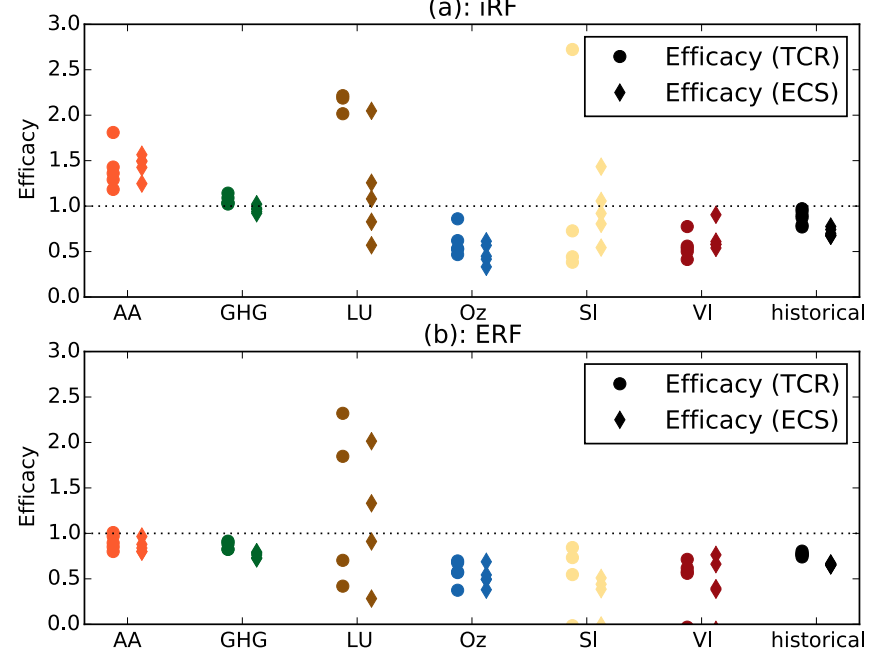

Figure S2: Transient (circles) and equilibrium efficacies calculated for each ensemble member in the single-forcing and historical ensembles. Efficacies are calculated using (a) iRF and (b) ERF. 


\begin{tabular}{|l||l|l||l|l|}
\hline \multicolumn{1}{|c||}{} & \multicolumn{2}{l|}{ Instantaneous RF (iRF) } & \multicolumn{2}{l|}{ Effective RF (ERF) } \\
\cline { 2 - 5 } & $E_{\text {transient }}$ & $E_{\text {equilibrium }}$ & $E_{\text {transient }}$ & $E_{\text {equilibrium }}$ \\
\hline \hline $\mathrm{AA}$ & $1.42(0.96,1.87)$ & $\mathbf{1 . 4 5}(1.21,1.68)$ & $\mathbf{0 . 8 3}(0.69,0.98)$ & $0.93(0.78,1.07)$ \\
$\mathrm{LU}$ & $3.89(-2.20,9.98)$ & $1.16(0.08,2.23)$ & $1.81(-1.08,4.71)$ & $0.11(-4.81,5.04)$ \\
$\mathrm{Oz}$ & $\mathbf{0 . 6}(0.31,0.90)$ & $\mathbf{0 . 4 8}(0.26,0.69)$ & $\mathbf{0 . 5 3}(0.31,0.76)$ & $\mathbf{0 . 5 6}(0.33,0.79)$ \\
$\mathrm{SI}$ & $1.53(-1.16,4.22)$ & $0.95(0.32,1.58)$ & $0.35(-0.50,1.19)$ & $\mathbf{0 . 2 6}(-0.35,0.86)$ \\
$\mathrm{VI}$ & $\mathbf{0 . 5 6}(0.30,0.81)$ & $\mathbf{0 . 6 4}(0.36,0.93)$ & $\mathbf{0 . 4 5}(-0.07,0.98)$ & $0.47(-0.20,1.13)$ \\
historical & $0.87(0.73,1.02)$ & $\mathbf{0 . 7 1}(0.63,0.78)$ & $\mathbf{0 . 7 1}(0.67,0.75)$ & $\mathbf{0 . 7 1}(0.69,0.72)$ \\
\hline
\end{tabular}

Table S1: Transient and equilibrium efficacies (mean and 5-95\% confidence intervals) calculated from instantaneous (iRF) and effective (ERF) radiative forcings. Values significantly different from unity are in bold.

Table S1 lists the transient and equilibrium efficacies calculated from the GISS-E2-R single-forcing runs, along with uncertainties derived from the 5member ensembles for each forcing.

We expect the transient and equilibrium efficacies of GHGs to be close to unity, as GHG forcing is dominated by $\mathrm{CO}_{2}$ forcing. However, the GHG-only simulations also contain methane, CFCs, and other greenhouse gases, which may cause the efficacy to differ from one [4]. Additionally, different manifestations of internal variability, damped somewhat in the 5-member ensemble averages, result in GHG TCR and ECS values that depart from $1.4^{\circ} \mathrm{C}$ and $2.3^{\circ} \mathrm{C}$, respectively. Finally, these deviations from published TCR/ECS values reflect the role of ocean heat uptake efficacy: as the pattern of surface warming evolves, outgoing flux into space, and thus the rate at which heat must be taken up by the deep ocean, changes.

Figure S4 shows the GHG-only TCR and ECS estimated from decadal mean temperature, forcing, and $\mathrm{OHC}$ changes as a function of time. The spread determined by the individual ensemble members becomes smaller toward the end of the historical record as the forcing grows stronger, but the ECS and TCR ranges encompass the $\mathrm{CO}_{2}$-only values. Rather than assume the efficacy of GHGs to be 1 , we estimate GHG efficacy and its uncertainty from the ensemble. 
(a): TCR

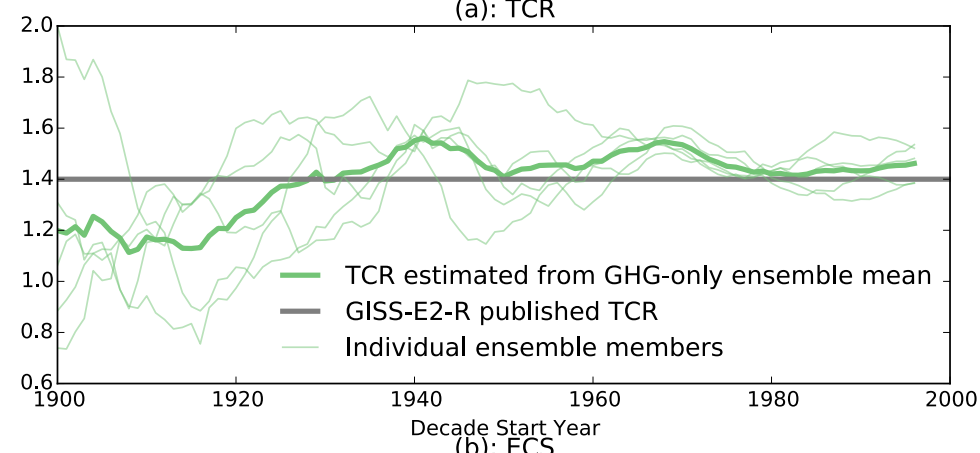

(b): : ECS

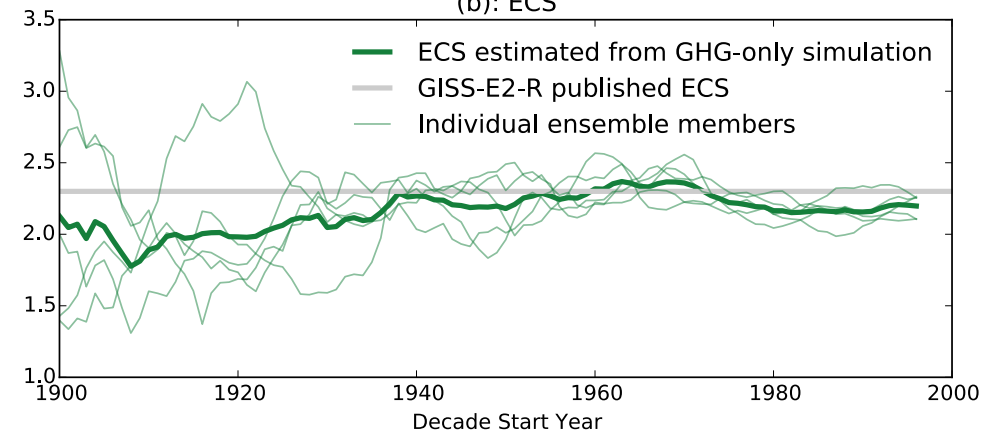

Figure S3: (a): Transient and (b): equilibrium sensitivities estimated from 10year means of temperature and forcing change and $\mathrm{OHC}$ change (in the equilibrium case) relative to pre-industrial control values. 


\begin{tabular}{|c|c|c|c|c|c|c|c|c|}
\hline Reference & $\Delta T$ & $\Delta Q$ & $F_{G H G}$ & $F_{A A}$ & $F_{L U}$ & $F_{O z}$ & $F_{S l}$ & $F_{V l}$ \\
\hline S14 & $0.68 \pm 0.1$ & $0.55 \pm 0.27$ & $2.47 \pm 0.12$ & $-0.825(+0.3,-0.5)$ & $-0.085 \pm 0.085$ & $0.27 \pm 0.14$ & $0.03 \pm 0.05$ & $-0.125 \pm 0.035$ \\
\hline LC14 & $0.71 \pm 0.15$ & $0.36 \pm 0.27$ & $2.37 \pm 0.57$ & $-0.68(+0.8,-0.1)$ & $-0.1025 \pm 0.1$ & $0.27 \pm 0.21$ & $0.03 \pm 0.05$ & $0.0 \pm 0.04$ \\
\hline O13 & $0.75 \pm 0.1$ & $0.65 \pm 0.27$ & $2.4 \pm 0.28$ & $-0.7 \pm 0.7$ & $-0.15 \pm 0.1$ & $0.28 \pm 0.17$ & $0.05 \pm 0.05$ & $-0.12 \pm 0.04$ \\
\hline
\end{tabular}

Table S2: Observational estimates from three references: S14[13], LC14[7], and O13[10]. All forcings and OHC uptake rates are in units of watts per square meter; temperature $\Delta T$ is in $\mathrm{K}$.

\section{Observations}

Following previous work[10], we use the HadCRUT4 estimate[9] of the 20002009 temperature change relative to the base period (1860-1879), yielding $\Delta T=$ $0.75 \pm 0.02{ }^{\circ} \mathrm{C}$. For radiative forcing, we use IPCC best estimates and uncertainties of the effective radiative forcing (ERF) due to aerosols, solar and volcanic forcing, well-mixed greenhouse gases, ozone, and land use changes from 2000-2009 relative to the base period. There is some ambiguity in these forcing definitions ${ }^{1}$, and for completeness we will investigate the implications of efficacies calculated using both iRF and ERF.

In Figure S3, we illustrate how existing TCR/ECS calculations using combined forcings are modified when efficacies (calculated in the GISS perfect model framework) are taken into account. We rely on three estimates[10, 13, 7], hereafter 013, S14, and LC14. Differences between our median estimates and confidence intervals and the previously reported estimates likely result from our treatment of forcing uncertainties. LC14 and $\mathrm{O} 13$ use the total radiative forcing in order to estimate sensitivities; here we attempt to break down this total forcing into a sum of contributions from individual forcing components, and treat uncertainties in these individual forcings as independent. The values of relevant quantities are shown in Table S2. In 013, present-day forcing estimates are defined as 20002009 averages with respect to the $1860-1879$ base period. We estimate these forcings from the values and uncertainties given in IPCC AR5 WG1 Table All.1.2, Table 8.SM.5, and Table 8.6. The IPCC report lists 2011 forcing uncertainties, which we scale by the ratio of 2009 forcing to 2011 forcing. We use ocean heat content uptake rate and temperature change values reported in the 013 supplementary material. The values used in LC14 are similar, although forcings are defined as 1995-2011 averages with respect to a base period of 1959-1882. We also use their different, lower values of ocean heat uptake rate and their stated

\footnotetext{
${ }^{1}$ For example, the best-estimate $1750-2011$ iRF and ERF values given by the IPCC are identical, except for aerosols (Table 8.SM.5 and Table 8.6).
} 


\begin{tabular}{|c|c|c|c|c|c|c|c|c|c|}
\hline Percentile & $\mathrm{S} 14(\mathrm{E}=1)$ & S14 (iRF) & S14 (ERF) & LC14 $(\mathrm{E}=1)$ & LC14 (iRF) & LC14 (ERF) & $\mathrm{O} 13(\mathrm{E}=1)$ & O13 (iRF) & O13 (ERF) \\
\hline TCR (median) & 1.4 & 2.0 & 1.9 & 1.3 & 1.6 & 1.7 & 1.3 & 1.8 & 1.8 \\
\hline $5 \%$ & 1.0 & 1.3 & 1.3 & 0.9 & 0.8 & 1.1 & 0.9 & 1.0 & 1.1 \\
\hline $17 \%$ & 1.2 & 1.5 & 1.5 & 1.0 & 1.1 & 1.3 & 1.0 & 1.2 & 1.4 \\
\hline $83 \%$ & 1.7 & 2.7 & 2.3 & 1.7 & 2.7 & 2.4 & 1.8 & 3.1 & 2.6 \\
\hline $95 \%$ & 2.0 & 3.6 & 2.7 & 2.4 & 5.5 & 3.9 & 2.3 & 5.9 & 3.6 \\
\hline ECS (median) & 2.1 & 4.0 & 3.6 & 1.5 & 2.0 & 2.3 & 2.0 & 2.9 & 3.4 \\
\hline $5 \%$ & 1.4 & 2.1 & 2.2 & 1.0 & -3.8 & 1.3 & 1.1 & -14.1 & 1.4 \\
\hline $17 \%$ & 1.7 & 2.7 & 2.7 & 1.2 & 1.4 & 1.7 & 1.4 & 1.5 & 2.2 \\
\hline $83 \%$ & 2.7 & 6.7 & 5.3 & 2.2 & 3.8 & 3.8 & 3.2 & 6.9 & 6.8 \\
\hline $95 \%$ & 3.4 & 12.6 & 7.9 & 3.6 & 8.8 & 7.2 & 5.4 & 19.4 & 15.8 \\
\hline
\end{tabular}

Table S3: TCR and ECS percentiles calculated using observational estimates from three references: S14[13], LC14[7], and O13[10] assuming unit efficacy $(E=1)$ and efficacies calculated from instantaneous (iRF) and effective (ERF) radiative forcing.

temperature change. In S14, the responses to the sum of CMIP5 aerosol, ozone, and land use forcings are estimated using the differences between historical and the sum of "historicalNat" and "historicalGHG" simulations, with forcings and uncertainties determined from ACCMIP data.

In calculating TCR and ECS from these forcing, temperature, and ocean heat uptake values, we draw samples from normal distributions in the case where uncertainty is taken to be symmetric about the mean. Where the uncertainties are not symmetric about the mean (e.g. anthropogenic aerosol forcings) samples are drawn from a lognormal distribution.

Means, medians, and confidence intervals for TCR and ECS derived from these observational estimates are shown in Table S3.

\subsection{Incorporating estimated efficacies}

In order to take forcing efficacy into account, we scale each observed or simulated forcing $\Delta F_{i}$ by the calculated efficacy $E_{i}$. This modifies $\mathrm{Eq}(1)$ in the main text:

$$
\sum_{i}^{n_{\text {forcings }}} E_{i} \Delta F_{i}=\lambda_{T C R} \Delta T \quad ; \quad \sum_{i}^{n_{\text {forcings }}} E_{i} \Delta F_{i}=\lambda_{E C S} \Delta T+\Delta Q .
$$

In the perfect model framework, this results in a revision of the best guess TCR (calculated from the sum of single-forcing experiments) from $1.0^{\circ} \mathrm{C}$ to $1.4^{\circ} \mathrm{C}$ and of ECS from $1.4^{\circ} \mathrm{C}$ to $2.4^{\circ} \mathrm{C}$ (Figure S4). 
(a): TCR

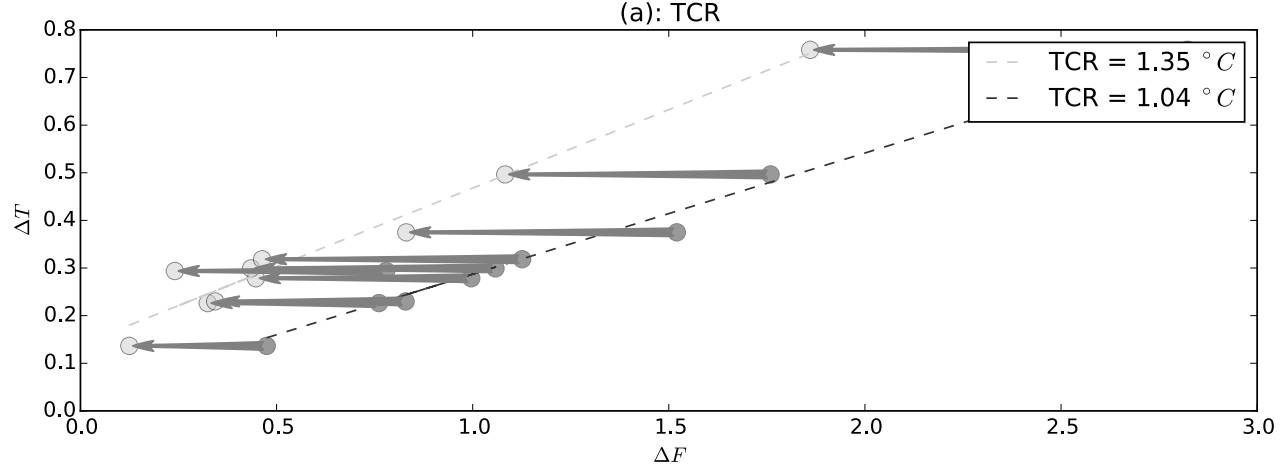

(b): ECS

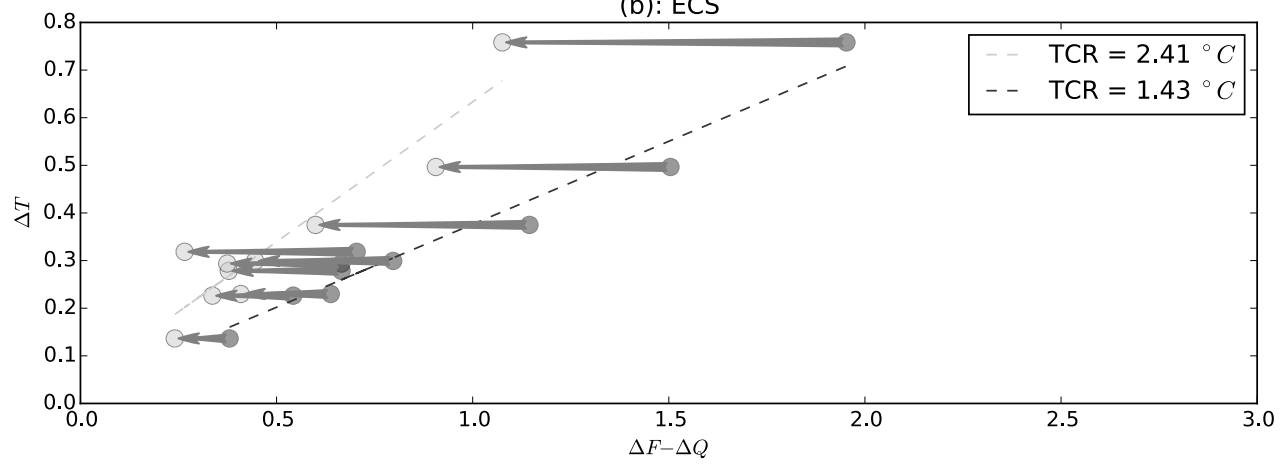

Figure S4: (a): Transient and (b): equilibrium sensitivities estimated from 10year means without (dark gray) and with (light gray) efficacy scaling. 


\section{References}

[1] K. C. Armour, C. M. Bitz, and G. H. Roe. Time-varying climate sensitivity from regional feedbacks. J. Clim., 26:4518-4534, 2013.

[2] J. M. Gregory, W. J. Ingram, M. A. Palmer, G. S. Jones, P. A. Stott, R. B. Thorpe, J. A. Lowe, T. C. Johns, and K. D. Williams. A new method for diagnosing radiative forcing and climate sensitivity. Geophys. Res. Lett., 31, 2004.

[3] JM Gregory and PM Forster. Transient climate response estimated from radiative forcing and observed temperature change. Journal of Geophysical Research: Atmospheres (1984-2012), 113(D23), 2008.

[4] J. E. Hansen, M. Sato, R. Ruedy, L. Nazarenko, A. Lacis, G. A. Schmidt, G. Russell, I. Aleinov, M. Bauer, S. Bauer, N. Bell, B. Cairns, V. Canuto, M. Chandler, Y. Cheng, A. Del Genio, G. Faluvegi, E. Fleming, A. Friend, T. Hall, C. Jackman, M. Kelley, N. Y. Kiang, D. Koch, J. Lean, J. Lerner, K. Lo, S. Menon, R. L. Miller, P. Minnis, T. Novakov, V. Oinas, Ja. Perlwitz, Ju. Perlwitz, D. Rind, A. Romanou, D. Shindell, P. Stone, S. Sun, N. Tausnev, D. Thresher, B. Wielicki, T. Wong, M. Yao, , and S. Zhang. Efficacy of climate forcings. J. Geophys. Res., 110, 2005.

[5] Isaac M Held, Michael Winton, Ken Takahashi, Thomas Delworth, Fanrong Zeng, and Geoffrey K Vallis. Probing the fast and slow components of global warming by returning abruptly to preindustrial forcing. Journal of Climate, 23(9):2418-2427, 2010.

[6] J. T. Houghton, editor. Climate Change: the IPCC Scientific Assessment. Cambridge University Press, 2001. Report by the Intergovernmental Panel on Climate Change.

[7] Nicholas Lewis and Judith A. Curry. The implications for climate sensitivity of AR5 forcing and heat uptake estimates. Clim. Dyn., sep 2014.

[8] R. L. Miller, G. A. Schmidt, L. S. Nazarenko, N. Tausnev, R. Ruedy, M. Kelley, K. K. Lo, I. Aleinov, M. Bauer, S. Bauer, R. Bleck, V. Canuto, Y. Cheng, T. L. Clune, A. Del Genio, G. Faluvegi, J. E. Hansen, R. J. Healy, N. Y. Kiang, D. Koch, A. A. Lacis, A. N. LeGrande, J. Lerner, S. Menon, V. Oinas, J. Perlwitz, M. J. Puma, D. Rind, A. Romanou, G. L. Russell, M. Sato, D. T. 
Shindell, S. Sun, K. Tsigaridis, N. Unger, A. Voulgarakis, M.-S. Yao, and J. Zhang. CMIP5 historical simulations (1850-2012) with GISS ModelE2. J. Adv. Model. Earth Syst., 6:441-477, 2014.

[9] Colin P. Morice, John J. Kennedy, Nick A. Rayner, and Phil D. Jones. Quantifying uncertainties in global and regional temperature change using an ensemble of observational estimates: The HadCRUT4 data set. J. Geophys. Res., 117(D8), 2012.

[10] A. Otto, F. E. L. Otto, O. Boucher, J. Church, G. Hegerl, P. M. Forster, N. P. Gillett, J. Gregory, G. C. Johnson, R. Knutti, N. Lewis, U. Lohmann, J. Marotzke, G. Myhre, D. T. Shindell, B. Stevens, and M. R. Allen. Energy budget constraints on climate response. Nature Geosci., 6:415-416, 2013.

[11] Matthew D Palmer, Douglas J McNeall, and Nick J Dunstone. Importance of the deep ocean for estimating decadal changes in earth's radiation balance. Geophysical Research Letters, 38(13), 2011.

[12] Gavin A. Schmidt, Max Kelley, Larissa Nazarenko, Reto Ruedy, Gary L. Russell, Igor Aleinov, Mike Bauer, Susanne Bauer, M. K. Bhat, Rainer Bleck, Vittorio Canuto, Y. Chen, Ye Cheng, Thomas L. Clune, Anthony Del Genio, R. de Fainchtein, Greg Faluvegi, James E. Hansen, Richard J. Healy, Nancy Y. Kiang, Dorothy Koch, Andy A. Lacis, Allegra N. LeGrande, Jean Lerner, Ken K. Lo, Elaine E. Matthews, Surabi Menon, Ron L. Miller, Valdar Oinas, A. O. Oloso, Jan Perlwitz, Michael J. Puma, William M. Putman, David Rind, Anastasia Romanou, Makiko Sato, Drew T. Shindell, Shan Sun, R.A. Syed, Nick Tausnev, K. Tsigaridis, Nadine Unger, A. Voulgarakis, MaoSung Yao, and Jinlun Zhang. Configuration and assessment of the GISS ModelE2 contributions to the CMIP5 archive. J. Adv. Model. Earth Syst., 6:141-184, 2014.

[13] Drew T. Shindell. Inhomogeneous forcing and transient climate sensitivity. Nature Climate Change, 4(4):274-277, mar 2014.

[14] Michael Winton, Ken Takahashi, and Isaac M Held. Importance of ocean heat uptake efficacy to transient climate change. Journal of Climate, 23(9):2333-2344, 2010. 